\title{
LE DONNE NEL PANORAMA SANITARIO DEL TARDIO MEDIOEVO IN ITALIA
}

\author{
WOMEN IN THE HEALTHCARE PANORAMA OF THE LATE MIDDLE AGES IN \\ ITALY
}

Sabrina Veneziani

Università degli Studi di Bari

\section{Riassunto:}

Nella società europea medievale, la donna è sempre stata tradizionalmente esclusa dalla sfera pubblica e dalla possibilità di poter esercitare una professione liberale. La donna veniva ritenuta incostante e incapace di svolgere attività intellettuali alla pari degli uomini. Tuttavia a partire dal XII secolo si inizia a registrare in Europa una leggera apertura per le figure femminili in ambito professionale. In particolare esistono numerose testimonianze che mostrano come ad alcune donne venne riconosciuta la possibilità di esercitare la professione medica. Si tratta di un fenomeno assolutamente unico e che non ha ulteriori riscontri dopo il XV secolo. Nel seguente studio si cercherà di far luce sul contesto societario e sulle condizioni che resero possibili in alcuni casi l'esistenza della figura della donna-medico.

\section{Parole chiave:}

donne, panorama sanitario, professione

\section{Abstract:}

In medieval European society, women have traditionally been excluded from the public sphere and from the possibility of being able to exercise a liberal profession. The woman was considered inconstant and unable to carry out intellectual activities on a par with men. However, starting from the 12th century, a slight opening for female figures in the professional sphere began to be registered in Europe. In particular, there are numerous testimonies that show how some women were recognized the possibility of practicing the medical profession. It is an absolutely unique phenomenon that has no further evidence after the 15th century. The following study will try to shed light on the corporate context and on the conditions that made it possible in some cases the existence of the female doctor.

\section{Keywords:}

woman, healthcare landscape, medical profession 
"Lo duca e io per quel cammino ascoso intrammo a ritornar nel chiaro mondo; e sanza cura aver d'alcun riposo, salimmo sù, el primo e io secondo, tanto ch'i' vidi de le cose belle che porta ' 1 ciel, per un pertugio tondo. E quindi uscimmo a riveder le stelle".

(Dante Alighieri)

Considerata lo sterile corridoio tra la razionalità classica e la rinnovata fiducia rinascimentale, l'Età di Mezzo è stata lungamente investita di una reputazione nefasta ed acritica, con l'implicazione di un arco di tempo estesissimo ed un'area geografica estremamente ampia. Il ritrovato approccio alle umane scienze, trasmesse da eredità araba, e la ricerca di un sapere in letargo, attraverso nuove traduzioni latine dei classici greci, intrisero la cultura dell'uomo europeo medievale, soprattutto a partire dall' $X \mathrm{I}$ secolo.

Ma se per l'uomo del tardo Medioevo si schiudeva l'affascinante carotaggio dell'universo e della natura umana, alla donna la dimensione pubblica era negata: le foeminae non governavano, non ricoprivano cariche pubbliche, non incedevano nei tribunali, se non in veste di testimoni o accusatrici, non commentavano, non insegnavano e non potevano gestire il potere, tranne che nelle badie.

"Mulier enim nec praedicare nec docere potest: quia hoc officium exstraneum est mulieribus, nec sacra vasa contingere...nec iudicare." ${ }^{11}$ (cap. Non quaedam, X.5.38.10, del diritto canonico, alla glossa Praedicare)

L'occidente medievale incriminò la donna, in accordo con la tradizione aristotelica, di fragilità, debolezza, imperfezione, eccesso di umidità, mollezza e mobilità: “l'anima segue la costituzione del corpo, le donne hanno un corpo molle e instabile, le donne sono instabili e molli nella volontà e nel desiderio". (Egidio Romano: 272).

Umile costola di derivazione, ella è uomo mancato e imperfetto, pertanto inferiore. “Dio creò l'uomo simile a sé .... lo creò a immagine di Dio .... maschio e femmina li creò" recita la Genesi I, 26-27. I Padri della Chiesa girarono pagina e cambiarono capitolo, opponendo e diffondendo una citazione ben preferibile per l'epoca, Genesi II, 21-24: "Allora Dio (...) gli tolse una costola e richiuse la carne al suo posto. Con quella costola Dio, il Signore, formò la donna e la condusse all'uomo". Sulla propria pelle, la donna sconterà questo abile colpo di mano perpetrato dai teologi, che al peccato originale sostituirono un peccato sessuale.

1 Trad.: “ la donna infatti non può predicare né insegnare: poiché questo è un compito estraneo alle donne, né toccare gli arredi sacri...né giudicare". 
Relegate tra mura monastiche o domestiche, alle mulieres dell'Evo Medio era negata l'esegesi, era vietata la predicazione, impedito il magistero.

"nam in collegio decurionum non posset esse mulier... vel in collegio doctorum... eis docendi et iudicandi officium interdictum est". ${ }^{2}$ (Bartolo, n. 16)

Discriminate ed emarginate, "non dovevano imparare né a leggere né a scrivere, se non per diventar monaca, perché dal leggere e dallo scrivere delle donne molti mali sono venuti". (Filippo da Novara: 16) Le donne non possedevano i quattro requisiti fondamentali per insegnare dedotti dalla dottrina: costanza, efficacia, autorità, vivacità; e si insiste ancora nel Seicento: si enim loquitur, magis incitat ad luxuriam et irritatur. ${ }^{3}$ (Brancaccini F.D., lib I, cap. XVII, n.15)

Una delle ragioni di cotanta inferiorità del troppo gentil sesso è da ascrivere anche alla mensile perdita di sangue mestruale. La trasgressione al veto ecclesiastico, posto ai coniugi di effondersi in presenza di flusso mestruale, sarebbe stata punita con la nascita di figli affetti da lebbra, la malattia dei secoli bui.

"Con dolore partorirai" dice il Signore ad Eva; "con il sudore del tuo volto mangerai il pane" intima ad Adamo". All'uomo il labor, alla donna il dolor.

Una Rinascenza si compì a partire dal XII secolo. In Europa si registrò un sensibile sviluppo economico, a cui si accompagnò un riaffermarsi dell'individuo. Una riorganizzazione degli assetti lavorativi fu la naturale conseguenza delle mutate condizioni; negli ingranaggi dei sistemi produttivi fu integrato un numero sempre maggiore di donne. Era la coppia e non più il solo marito ad occuparsi di aziende, cittadine o rurali, a carattere sempre più familiare e autosufficiente. Specifici ambiti di competenza operativa maschili e femminili si enuclearono rapidamente.

Nonostante il minorita Andrea di Regensburg, con manifesta acrimonia, avesse paragonato le aspirazioni imprenditoriali muliebri ad un superfluo volo di galline al di là dello steccato (cit. da Dienst, 1984), signore e signorine del tardo Medioevo ebbero l'improntitudine di affermarsi attivamente extra moenia familiae. Quando il mondo era più giovane di otto secoli, nello spazio aperto in Europa fra il XII e XIII secolo si affacciarono silenziosamente sparute donne in carriera.

Le mulieres, quindi, necessitarono di un'istruzione basica e si formarono soprattutto in casa, in famiglia o con precettori privati. Già alla fine del XIII secolo, alcune città europee, Londra e Parigi, potevano vantare la nascita di scuole elementari femminili, in cui la docenza era affidata a maestre e donne dirigenti necessariamente sposate. Una brevissima finestra storica, nella prima metà del XIV secolo, può annoverare persino la

2 Trad.: “Infatti, una donna non può stare nel collegio dei decurioni...o nel collegio dei dottori...a loro è interdetto il compito di insegnare e giudicare".

3 Trad.: "se parla infatti, incita di più alla lussuria e si eccita". 
promiscuità scolastica; immediatamente bandita, per il ripristino di una più dignitosa separazione dei sessi (Uitz, 1988: 130 e ss)

Ciò nonostante, le donne furono generalmente escluse dagli Studi Generali o Università.

1. “possint Et VIR ET FOemina MEdici ESSE"

L'atteggiamento antifemminista rimase invariato per tutto l'antico regime, come reliquato della tradizione romana e clericale: "omnes foeminae remotae sunt non tantum ab officiis publicis, sed et omnibus quae mentis acumen requirunt" . ${ }^{4}$ (Tiraqueau, cap. XXXI, n. 321)

Impensabile il riconoscimento formale dei titoli accademici; le donne operarono quali "professioniste", senza addottorarsi. (Di Noto Marrella, 1994: 120 e ss) Tuttavia, nessun ordinamento universitario escludeva esplicitamente le donne dagli studi superiori. Le Costituzioni di Federico II accettavano solo implicitamente' le donne alle Università di Salerno e Napoli. Il Re di Francia Giovanni, invece, nel 1352 emanò un editto che concedeva tutte le facilitazioni affinché "quam plurimi, utriusque sexus, mulieresque aliquae et vetulae, venientes ad villam Parisiensem, gratia praticandi" ${ }^{5}$ trovassero tutto il necessario per il proprio sostentamento per frequentare lo Studium di Parigi (du Boulay: tomo IV, 672). Con regale auspicio, Edoardo di Inghilterra promulgò delle leggi che nel modo più esplicito ammettevano che "possint et vir et foemina medici esse $^{6 \prime \prime}$ (Westfal, 1754).

Ma per le difficoltà poste alle signore di perseguire un regolare curriculum studiorum, la frequenza universitaria fu un ufficio del tutto virile.

Con somma fortuna per le donne della tarda Età di Mezzo, la formazione medica non rimase strettamente confinata tra le mura universitarie. Chiunque avesse superato con successo un esame davanti ad una Commissione apposita presieduta dal Priore del Collegio, ove questo esisteva, poteva praticare l'arte ippocratica.

"Women as well as men practiced medicine and surgery; as with their predecessors in the Roman empire, women's practice was limited neither to obstetrical cases nor to female patients. For example, the names of 24 women described as surgeons in Naples between 1273 and 1410 are known, and references have been found to 15 women practitioners, most of them Jewish and none described as midwives, in Frankfurt between 1387 and 1497..." (Siraisi, 1990: 27)

4 Trad.: "Tutte le donne sono lontane non solo dagli impieghi pubblici, ma da tutte quelle situazioni che richiedono una certa acutezza della mente".

5 Trad.: "moltissimi, di entrambi i sessi, anche donne e anziane, venendo alla città di Parigi, per poter praticare".

6 Trad.: "possano l'uomo e la donna essere medici". 
In ogni caso, un numero estremamente ristretto di donne nel Medioevo potette fregiarsi del titolo di 'medica'. Con il diffondersi delle scuole mediche, dal 1300 in poi, l'esercizio della medicina, almeno nella sua forma ufficiale, venne fatto dipendere, se non dal crisma di uno Studio Generale, almeno dal possesso di una licenza. Le signore non si permisero di frequentare regolari corsi di studi universitari e raramente misero in discussione la propria esperienza davanti ad una Commissione, formata da soli uomini, per ottenere l'agognata autorizzazione. Praticarono piuttosto la medicina e la chirurgia come 'empiriche' (termine ampiamente utilizzato dai doctores artium et medicinae per riferirsi ai professionisti della salute senza titoli accademici) detentrici però di un sapere ereditato da tradizione familiare o coniugale; in nulla differenti dai loro colleghi maschi.

Diffuso durante tutto l'Evo Medio, l'insegnamento privato costituì il sistema più comune di erudizione e apprendimento. Nei centri urbani più importanti fiorirono Scuole di Maestri privati, che insegnarono a gruppi di allievi la medicina pratica; trasmettendo nella più totale confidenza da consorteria privilegiata metodi terapeutici, ricette e sistemi diagnostici, messi a punto personalmente. Segreti professionali, che nulla avevano a che vedere con la deontologia corrente, si tramandavano gelosamente da maestro ad allievo, da padre a figlio o figlia, e da marito a moglie. In tal maniera, facilmente si strutturarono intere dinastie mediche. Una moglie o una figlia, in assenza di eredi maschi, si faceva carico di raccogliere l'eredità dell'esercizio praticato dal congiunto ritiratosi, tentando di rendersi abile nella stessa specialità e di mantenere la medesima cerchia clientelare.

Non si trattava assolutamente di volgari ciarlatane o fattucchiere praticone, nemmeno di semplici levatrici o mammane che assistevano le donne al momento del parto e si occupavano dei disordini del sistema riproduttore, non essendo permesso ai maschi l'approccio alle pudenda femminili. Già Sorano di Efeso esplicita: "quid est obstetrix? Femina omnium muliebrium causarum docta, etiam medicinali exercitatione perita", sottolineando l'ampia casistica terapeutica di cui l'ostetrica doveva occuparsi. (Valentin, 1882: 6) Le donne-medico nel Tardo Medioevo erano specialiste.

Come ben sottolinea M. Green (1989), non esiste ancora una letteratura sistematica sulla pratica della medicina da parte delle donne nel Medioevo, almeno per quel che riguarda l'Italia; ma una serie di studi localistici, e purtroppo abbastanza dispersi, hanno fornito testimonianze delle abilità mediche muliebri e della loro presenza sul territorio.

Una visibilità tutta diversa è stata concessa alle Mulieres Salernitanae; signore che furono ammesse a frequentare la Scuola Medica Salernitana, e che in alcuni casi ne divennero docenti, i cui nomi echeggiano altisonanti nella pagine dei testi di Storia della 
Medicina: Trotula de' Ruggiero, Abella di Castellomata, Rebecca Guarna, Mercuriade, Costanza Calenda, et alterae. (De Renzi, 1852-59: vol. 1., 159-160)

Il ricchissimo Archivio Angioino ha traghettato una polposa documentazione sulla presenza di donne-medico tra il XIII e il XV secolo nel Regno di Napoli. Ben 24 nomi di chirurghe sono tornati alla luce. Tredici di esse possedevano una precisa licenza per praticare la chirurgia sulle donne ed occuparsi di precise questioni attinenti la ginecologia e le malattie delle mammelle. Sei sono catalogate nei documenti quali ydiotae, con esplicito riferimento all'incapacità a leggere il latino, se non addirittura il vernacolo. Nessuna è esplicitamente definita come litterata. ${ }^{7}$ (Calvanico, 1962)

Nel 1321, il Duca Carlo di Calabria conferì a Francesca, moglie di Matteo Romano, la Laurea in chirurgia. (Uitz, 1988: 68) Di una sanitaria ebrea riferisce Ch. Landau, una certa Pereira, che ottenne nel 1460 la licenza all'esercizio della medicina nel Regno. In considerazione del fatto che "ad mulieres curandis viris sunt foeminae aptiores", re Ladislao di Durazzo incaricò maestro Benedetto di Roma, giudeo, perché esaminasse le cognizioni mediche e terapeutiche di Donna Cusina di Filippo de Pastino, probabilmente ebrea anch'ella, e nel 1404 le conferì la licenza per la pratica della professione chirurgica a Cosenza. (Baroni, 1888: 23) $\mathrm{E}^{\prime}$ verosimile che medici e medichesse di religione ebraica, oltre ad essere esaminati, curassero solo i propri correligionari, salvo permessi speciali delle autorità ecclesiastiche che potevano estenderne il raggio d'azione alla popolazione cristiana.

"The kingdom of Naples was one of the few areas where regulation allowed women to practise" sostiene P. Skinner (1997: 90), sottolineando la quasi invisibilità delle professioniste della sanità nei documenti medievali: "The number of known female practitioners is small... and may be distorted by biases inherent in the source" replica V. Nutton. (1995: 170) Il regno di Napoli costituì senza dubbio un'oasi a sé stante per le mulieres nel panorama della medicina medievale, soprattutto per la presenza della Scuola Salernitana e per il clima interculturale e multietnico, che si respirava; tuttavia numerosi documenti d'archivio riportano, se non altro, i nomi di alcune terapeute, che praticarono sul territorio nazionale.

7 Nel lavoro di Calvanico possono essere identificati i seguenti nomi, accompagnati dai numeri di registrazione: Adelicia da Capua (3006); Bona di Guglielmo di Odorisio da Miglionico (3119); Clarice di Durisio da Foggia (3127); Costanza da Barletta (1168, 1209); Francesca, moglie di Matteo da Romano di Salerno, passata alla storia come S. Francesca Romana (1321-22, 1451, 1872, 1874); Francesca, moglie di Vestis (916); Gemma da Molfetta (1981); Isabella da Ocre (3195); Lauretta, moglie di Giovanni da Ponte da Saracena (1413, 2023, 20269; Letizia di Manso da Friano (3072); Mabilia di Scarpa da S. Maria (3327, 3371, 3406); Margherita di Napoli, da S. Maria (3534); Margherita de Ruga $(3572,3620)$; Margherita da Venosa (3226); Maria Gallicia (1165, 1234); Maria Incarnata (3571); Polisena de Troya $(3598,3610)$; Raymunda de Taberna (3643); Sabella di Ocro (o di Erro) (3071); Sibilla de Afflicto di Benevento (3407); Sibilia da S. Giovanni Rotondo (3227); Trotta di Troya (966); Venturella Consinata (o Cisinato) (1875); Vigorita da Rossano (3512). Sono noti, inoltre Cusina di Pastino (registro angioino n. 318 anni 1338-39 D, carta 90 retro) e Mobilia Scarpa (anno 1338). In Regesta Neapolitana si legge di una certa Anna, medica de Balusano (RN, doc.23, 923). 
K. Park (1985) ha ritrovato negli elenchi delle corporazioni fiorentine quattro nomi certi, e due probabili, di medichesse regolarmente immatricolate. L'inventario si allunga spaventosamente se si annoverano nel territorio fiorentino anche levatrici e "speziali", farmaciste; la "Matricola"8 dell'Arte dal 1356 al 1409 include i nomi di ben 40 donne: un lungo elenco comprendente spesso paternità o stato coniugale delle lavoratrici, quasi a volerne legittimare la tradizione professionale. (La Sorsa, 1907: 600)

L'esistenza di 550 professionisti della salute tra il 774 e il 1555, due dei quali con certezza mediche, nel territorio di Siena, è stata verificata da A. Garosi (1958). Balza agli occhi il nome di Monna Mita medicha, che per l'anno 1390 era in credito di sette soldi con il comune di Siena, per le proprie prestazioni sanitarie.

Ad una famiglia di medici senesi, i fratelli Pandolfo, Bernardo ed Agnese, nel XIII secolo era stata rilasciata una concessione per operare a Perugia ${ }^{9}$; un evento del tutto inconsueto e concesso solo raramente a professionisti di chiara fama.

Da Pistoia giunge memoria di una magistra chirurgiae anonima, che "medicò a Frate Roberto da Firenze lo capo" due volte, e venne ricompensata con sei lire e nove soldi a medicazione. Altre "donne tanto in Pistoia, che nelle campagne, che non si peritavano a farla da medichesse" circolavano comunque nel territorio della città, prestando i propri servizi per l'assistenza soprattutto alle donne. (Chiappelli, 1906)

Bologna 'la dotta' non può farsi vanto dello stesso curriculum di presenze femminili tra le fila dei propri medici nel Medioevo. Nel 1436, Dorotea Bocchi "instruttissima della più recondita Filosofia, ed in essa laureata" subentrò al padre, professore all' Alma Mater tra il 1390 e il 1436, e ne continuò la missione didattica, istruendo studenti, provenienti da ogni nazione d'Europa, per un profitto di cento lire. (Alidosi, 1664: 16) Con sicurezza si ricorda anche Jacobina, filia quondam Bartholomei, nell'anno 1304. (Sarti 1769: Tomo I, 436)

Mentre in Piemonte sono registrate solo due donne nell'ambiente sanitario del XIV secolo $^{10}$ (Malacarne), nel Veneto ne spicca un numero più cospicuo.

"Consultandi prudentia, medendique arte...rariori exemplo eminuit"11 la dottoressa Adelmonta Maltraversa a Padova, figlia di Bontraverso Maltraversa conte di Castronuovo e moglie di Jacobino da Carrara. (Rhodius: nota CXXII, 194)

8 La Matricola fa parte dell' Archivio dell'arte dei Medici e Speziali, e l'elenco dei nomi è contenuto nei volumi 7 e 9 .

9 Deliberazione presente nella rubrica CCLXXII dello Statuto riguardante i medici della città, dell'anno 1279, presente nell'Arch. di Stato di Perugia .

10 Ghilietta medica e Leonetta moglie di Giovanni da Gozzano.

11 Trad.: "per la prudenza nella consultazione...per la maestria nel medicare, per il raro esempio spicca" 
La Repubblica veneziana, nella tarda Età di Mezzo, ammetteva all'esercizio della professione chiunque avesse ottenuto licenza, superando l'esame davanti al Collegio, e coloro che, preceduti da una buona fama, "per grazia" ne avessero avuto la possibilità dal Maggior Consiglio, pur in assenza di titoli specifici.

Nella Serenissima operarono con certezza sei medichesse nel XIV secolo; a L. Münster (1954) se ne deve il rinvenimento nell'Archivio di Stato della città. Un intreccio di traversie giudiziarie si interseca con la vicenda personale della protagoniste. A Magistra Beatricae medicae, vedova del maestro Gherardo da Creta, fu comminata una sanzione di 15 lire piccole per aver accettato un anticipo sul compenso per una cura non ancora iniziata. Mentre Margherita, moglie del chirurgo Menego o Meneghello di S. Fosca, fu multata di 25 lire, essendosi macchiata di un crimine intollerabile: aver medicato molte persone senza autorizzazione; $\mathrm{fu}$, in seguito, assolta dal Maggior Consiglio e le fu conferita la licenza per esercitare liberamente. Altra licenza fu concessa a Draga, donna-medico di origine slava, in considerazione dei successi ottenuti nella dura della gotta e delle malattie oculari.

Vedova del Maestro Rainaldo medicus cirurgiae, Jacobina incorse nelle ire delle autorità giudiziarie per non aver denunciato un "percosso", a cui aveva prestato assistenza. Una vicenda conclusa felicemente poiché non vi era aliqua malicia nota nell'operato.

Accusata di aver insegnato ad una donna la cura di una particolare malattia oftalmologica, Lucia moglie di Giorgio di Santa Lucia dovette pagare un'ammenda di 20 soldi. Da ultimo è il caso di una 'medicatrice', che cioè lavorava clandestinamente, acciuffata per aver consegnato un unguento e condannata a versare 40 soldi.

Dopo la metà del XV secolo e fino all'epoca dei Lumi, in un panorama sanitario declinato al maschile, le donne-medico scomparvero, lasciando il posto alle sole levatrici; per un'assistenza tutta pratica fatta dalle donne per le donne.

\section{BIBLIOGRAFIA}

-ALIDOSI P., Catalogo di tutti i Dottori Collegiati in Filosofia e Medicina, Bologna, Tip. Monti, 1664.

-BARONI N., "Notizie raccolte da registri di Cancelleria del re Ladislao di Durazzo", in Arhivio Storico Napoletano, anno XII, fasc. I, 1888.

-BARTOLO DA SASSOFERRATO, In primam Digesti novi partem commentaria ... Necnon additioni. Petripauli Parisij, Venetiis, 1557. 1. Sodales (D.47.22.4)

-BRANCACCINI, D., Magistri fr. Dominici Mariae de Brancaccinis Florentini ... serenissimi Cosmi tertij Magni Etruriae de iure doctoratus libri 4. Romae, typis, \& sumptibus Nicolai Angeli Tinassij impressoris Cameralis, \& Vaticani, 1689. 
-BRUGARO A., "Contributi alla storia dei medici pisani dal XII secolo al XIV secolo", in Studi Storici, 1909, vol. XVIII, fasc II.

- CALVANICO, Raffaele. Fonti per la storia della medicina e della chirurgia per il regno di Napoli nel periodo angioino (a. 1273-1410), Napoli, L'Arte Tipografica, 1962, items 1413, 1451 e 1872, 3071 (cf. 3195), 3226, 3598, e 3643.

-CHIAPPELLI A., "Medici e Chirurghi in pistoia nel Medioevo", in Bullettino Storico Pistoiese, 1906, vol VIII, fasc 1-2 e 4; Vol IX, fasc 4; vol. X, fasc1-4.

-CONRAD L. I., Neve M., Nutton V., Porter R., Wear A., The western medical tradition :800 BC to AD 1800. Cambridge : Cambridge University Press, 1995.

-DANTE ALIGHIERI, Divina Commedia, Inferno, canto I.

-DE RENZI S., Collectio Salernitana, 5 voll, Napoli, Filatre-Sebezio, 1852-59.

-DIENSTH., "Rollenaspekte von Männern und Frauen im Mittelalter in Spätmittelalter”. In Weiblichkeit oder Feminismus? (Beiträge zur interdisziplinären Frauentagung Konstanz 1983) Claudia Opitz (a cura di), Weingarten, Drumlin, 1984, pp. 137-157.

-DI NOTO MARRELLA S., Doctores : contributo alla storia degli intellettuali nella dottrina del diritto comune. Padova, CEDAM, 1994.

-DU BOULAY C. E., Historia Universitatis Parisiensis, Paris, F. Noel et P. de Bresche, 1665-1673.

-EGIDIO ROMANO, de regimine principum libri III, apud Bartholomeaum Zanettum, Romae 1607.

-FILIPPO DA NOVARA, Les quatres âges de l'hommes, ed. M. de Fréville, Paris, 1888. -GAROSI A., Siena nella storia della medicina (1240-1555), Fizenze, Ed. L. Olschki, 1958.

-GREEN M., "Books as a Source of Medical Education for Women in the Middle Ages". In Dynamis, 2000, 20, 331-369.

(Notas)

1) GREEN M., "Women's Medical Practice and Health Care in Medieval Europe," Signs Vol. 14, Iss. 2 (Winter 1989); pg. 434-74.

-GREEN M., “Documenting medieval women's medical practice”. In Practical Medicine from Salerno to the Black Death. Roger French, Jon Arrizabalaga, Andrew Cunningham and Luis García-Ballester (a cura di), Cambridge : Cambridge University Press, 1994: 322-35.

- KRISTELLER P.O., “Learned women of Early Modern Italy: Humanist and university Scholars", in Beyond their sex: lernead women of the European Past, Ed. Patricia H. Labalm, NY, New York University Press, 1984. 
-LANDAU Ch., “Zur Geschichte der Juden in Italien”, in Frankfurter Zeitung, 1876.

-LA SORSA A., L'arte dei medici, speziali e merciai a Firenze e negli altri comuni italiani, Molfetta, 1907.

-MALACARNE V., Delle opere dei medici piemontesi prima del secolo XVI, nella Real Casa di Savoia", Torino, 1786.

- MÜNSTER L., “Womens doctors in Medieval Italy”, in Ciba Symposium (Ed. Inglese) 10, n.3, 1962, pp. 136-140.

- MÜNSTER L., “Notizie di alcune medichesse veneziane della prima metà del Trecento", in Scritti in onore del Prof. Pazzini, Saluzzo, Ed. Minerva Medica, 1954, pp. 180-187.

-PARK, Katharine. Doctors and Medicine in Early Renaissance Florence, Princeton, Princeton University Press, 1985, pp. 99-101.

-PLEIJ H.,"Arbeitsteilung in der Ehe. Literatur und soziale Wirklinchkeit im Spätmittelalter , , in Eheglück und Lebensjoch. Bilder von Liebe, Ehe und Familie in der Literatur des 15. und 16. Jahrhunderts, a cura di M.E. Müller, Weinheim u. Basel 1988, pp. 105-24.

-Regesta Neapolitana, in Monumenta ad Neapolitani Ducatus Historiam Pertinentia, ed. B. Capasso, IIi, Napoli 1885.

-RHODIUS I., ad Scribonium Largum, Edizione Patavina del 1655.

-SARTI P., De clariis archigymnasii Bon. Professoribus, Ediz. I, Bononiae, 1769.

-SIRAISI, N., Medieval \& early Renaissance medicine : an introduction to knowledge and practice. The Univ. of Chigaco Press, Chicago e Londra, 1990.

-SKINNER P., Health and medicine in early medieval southern Italy. Leiden [etc.], Brill, 1997.

-UITZ, E., Die Frau in der mittelalterlichen Stadt, Leipzig, Ed. Leipzig, 1988.

- VALENTIN R., Sorani Gynaeciorum vetus translatio latina, Leipzig, Ed. Teubner, 1882.

-WESTFAL A., De doctoratu mulierum, Gryphisfaldiae, 1754. 